\title{
Trabeculectomy with intraoperative retrobulbar triamcinolone acetonide
}

\author{
Malik Y Kahook' \\ Larissa Camejo ${ }^{2}$ \\ Robert J Noecker ${ }^{2}$ \\ 'Department of Ophthalmology, \\ The Rocky Mountain Lions Eye \\ Institute, University of Colorado \\ Health Sciences Center, Aurora, CO, \\ USA; ${ }^{2}$ UPMC Eye Center, Eye and Ear \\ Institute, University of Pittsburgh \\ School of Medicine, Pittsburgh, PA, USA
}

\begin{abstract}
Use of topical steroids is an important component of postoperative care after filtration surgery. Efficacy of postoperative medications is affected by patient adherence and physical limitations in the elderly population often prohibit proper dosing of ophthalmic drops. We describe a technique for the use of intraoperative retrobulbar triamcinolone acetonide in trabeculectomy surgery and report on postoperative outcomes. This technique appears safe and may be an attractive method of delivering a steroid depot at the time of trabeculectomy.
\end{abstract}

Keywords: glaucoma, trabeculectomy, steroids

\section{Introduction}

Antifibrotic medications augment the success of trabeculectomy procedures by decreasing fibroblast proliferation and scar formation. In particular, Mitomycin C (MMC) acts as an alkylating agent and inhibits DNA-dependent RNA synthesis leading to decreased fibroblast proliferation. MMC also has direct toxic effects on proliferating and nonproliferating cells causing cell apoptosis. ${ }^{1,2}$ Despite the improved success rate of antifibrotic-assisted trabeculectomy, many cases fail due to persistent inflammation, scar formation, and eventual loss of intraocular pressure (IOP) control. ${ }^{3}$

The use of steroid drops postoperatively has been shown to increase the success rate of trabeculectomy surgery. ${ }^{4}$ Using drops requires a great deal of patient cooperation and instillation can be difficult in those with already impaired vision and/or physical limitations. We performed consecutive trabeculectomy procedures with MMC and retrobulbar triamcinolone acetonide (RTA) in patients considered at high risk of failure due to previous unsuccessful glaucoma surgery.

\section{Methods}

This is a retrospective review of trabeculectomy procedures performed between July 2005 and January 2006 using intraoperative MMC and RTA. All surgeries were done using fornix based conjunctival incisions and $\mathrm{MMC}(0.2 \mathrm{mg} / \mathrm{ml}$ for 2 minutes $)$. RTA (20 $\mathrm{mg}$ in $0.5 \mathrm{ml}$ ) was deposited in the retrobulbar space using a blunt cannula attached to a $5 \mathrm{ml}$ syringe followed by conjunctival closure (Figures 1 and 2). Postoperative drop regimen consisted of prednisolone acetate $1 \%$, fourth generation fluoroquinolone, and atropine $1 \%$ each four times per day. Data collected included age, preoperative diagnosis, number of pre- and postoperative medications, pre- and postoperative IOP, and complication rates. Success was defined as IOP $>5$ and $<21 \mathrm{~mm} \mathrm{Hg}$ without need for further medications or surgical intervention. Analysis of variance was used to compare statistical significance between pre- and postoperative IOP values.

\section{Results}

Information was available from 14 consecutive cases, eight women and six men, undergoing trabeculectomy with MMC and RTA. Mean age was $66.93 \pm 14.51$ years with 


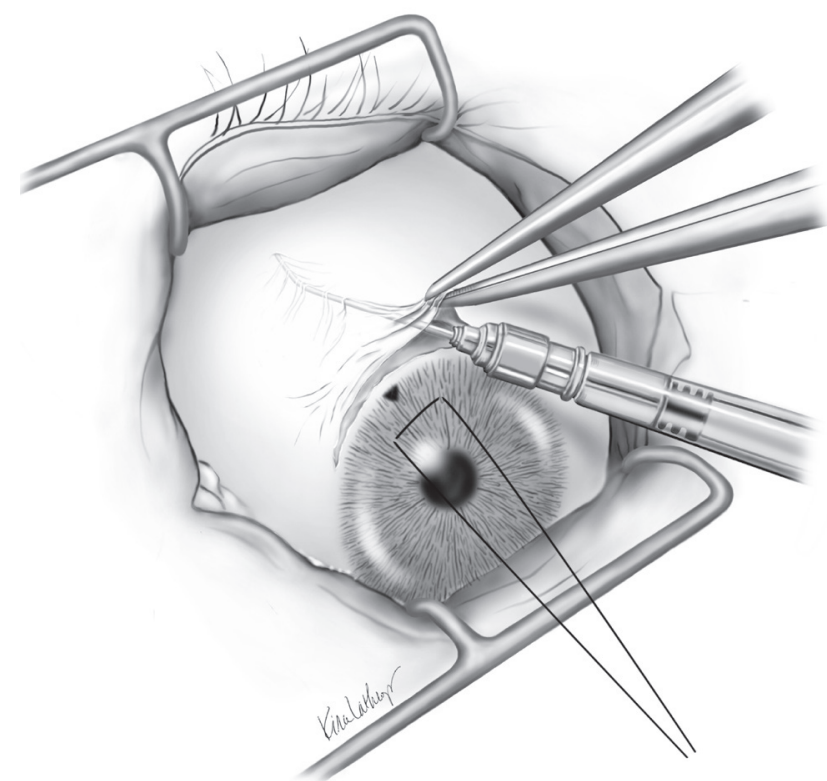

Figure I Triamcinolone acetonide is injected using a blunt cannula prior to conjunctival closure. The cannula is inserted in the hub and the steroid depot is injected in the retrobulbar space.

visual acuity ranging between 20/20 and 20/100. Preoperative diagnosis included 12 patients with primary open angle glaucoma, one with uveitic glaucoma, and one with congenital glaucoma. Thirteen patients had failed previous trabeculectomy with MMC and one had previous goniotomy at six months of age. Mean preoperative hypotensive drop use was $3.0 \pm 0.68$.

IOP decreased from a preoperative level of $26.43 \pm 8.32$ to $10.64 \pm 1.82$ at six months $(\mathrm{p}<0.001)$. The decrease in IOP was statistically significant at all time points (Table 1) and all patients remained off hypotensive drops for the duration of follow up. Three patients lost one line of vision attributed to cataract progression while the remaining 11 patients experienced no change in visual acuity. The patient with uveitic glaucoma was considered a treatment failure after IOP increased to $22 \mathrm{~mm} \mathrm{Hg}$ three months post-operatively. He required a bleb needling procedure with MMC and remains off hypotensive drops with an IOP of 8 after six months of follow-up. There were no cases of postoperative bleb leak, infection, hypotony or conjunctival ulceration.

\section{Discussion}

Pre- and postoperative use of steroids has been reported in patients undergoing trabeculectomy. ${ }^{4,5}$ Araujo and colleagues reported on the effects of postoperative corticosteroids on IOP and clinical course. ${ }^{4}$ They found that patients who received postoperative steroids were less likely to have higher IOP's,

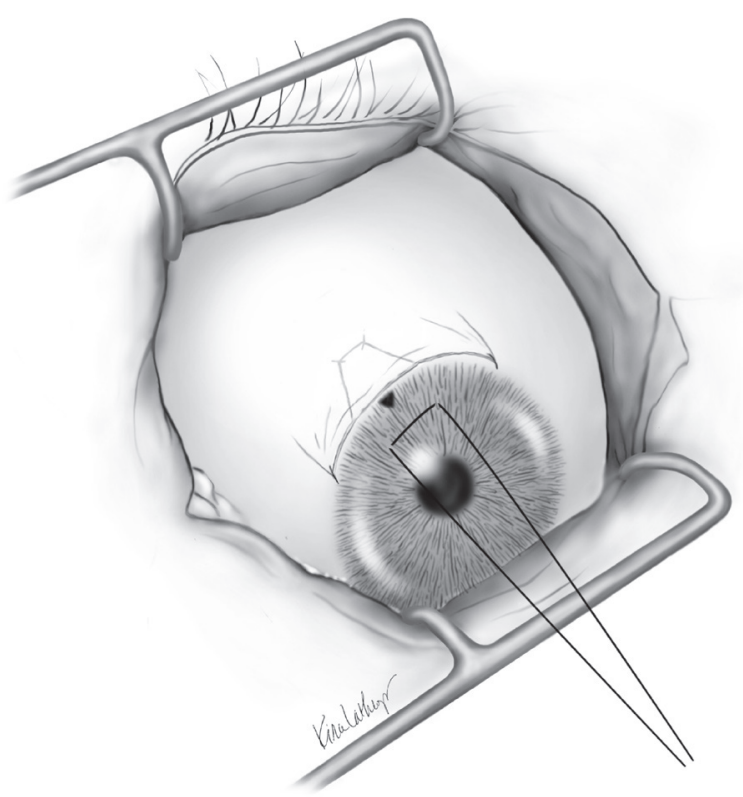

Figure 2 Two interrupted 9-0 vicryl sutures reapproximate the conjunctiva at the end of the case.

less likely to need further glaucoma surgery, and required less glaucoma medicines for IOP control. Giangiacomo and colleagues reported the use of subconjunctival triamcinolone acetonide injected prior to surgery in 15 eyes of patients considered to be at increased risk for postoperative scarring. ${ }^{5}$ All eyes were followed for six to 16 months with fourteen considered successfully controlled with an IOP of $12.4 \pm 4.6$ and only three of the 14 eyes required antiglaucoma medications. Tham and colleagues reported on the intraoperative subconjunctival injection of triamcinolone acetonide using a 27-gauge needle in 11 patients. Results showed this method to be compatible with successful clinical outcomes. ${ }^{6}$

Table I Pre- and postoperative intraocular pressure in patients receiving retrobulbar triamcinolone acetonide at time of trabeculectomy

\begin{tabular}{ll}
\hline IOP $(\mathbf{n}=\mathbf{1 4})$ & $\begin{array}{l}\text { Mean and standard } \\
\text { deviation }(\mathbf{m m ~} \mathbf{~ H g})\end{array}$ \\
\hline Preoperative IOP & $26.43 \pm 8.32$ \\
POD I IOP & $11.36 \pm 3.67$ \\
POD 7 IOP & $9.86 \pm 3.42$ \\
POD 30 IOP & $11.07 \pm 2.40$ \\
POD 90 IOP & $10.42 \pm 2.71$ \\
POD I80 IOP & $10.64 \pm 1.82$
\end{tabular}

Note: *Change in IOP from baseline was statistically significant $(P<0.001)$ at all time points.

Abbreviations: IOP, intraocular pressure; POD, postoperative day. 
The success rate, as defined in this study, was $93 \%$ (13/14 patients) with six months of follow-up. All patients experienced a decrease in IOP without the use of hypotensive glaucoma drops. The use of RTA in trabeculectomy with MMC appears safe in a patient population considered at high risk of failure and resulted in well-functioning diffuse blebs. The use of a cannula decreases the risk associated with use of a needle to inject steroids either pre-, peri-, or postoperatively and assures that steroids will reach the site of filtration independent of adherence to drop therapy. In contrast to injection of steroids adjacent to the bleb at the end of surgery using a needle, our technique allows for the medication to come in direct contact with the bleb surface without creation of an unnecessary conjunctival hole. Further studies will be needed, in a prospective and randomized fashion, to better delineate the utility of retrobulbar steroids combined with MMC in increasing the efficacy of trabeculectomy procedures.

\section{Acknowledgments}

We thank Kira L Lathrop MAMS for her contributions to Figures 1 and 2.

\section{Disclosure}

The authors have no competing interests or disclosures to report.

\section{References}

1. Chen CW. Enhanced intraocular pressure controlling effectiveness of trabeculectomy by local application of mitomycin-C. Trans Asia-Pacific Acad Ophthalmol. 1983;9:172.

2. Palmer SS. Mitomycin as adjunct chemotherapy with trabeculectomy. Ophthalmology. 1991;98:317-321.

3. Lama PJ, Fechtner RD. Antifibrotics and wound healing in glaucoma surgery. Surv Ophthalmol. 2003;48:314-346.

4. Araujo SV, Spaeth GL, Roth SM, et al. A ten-year follow-up on a prospective, randomized trial of postoperative corticosteroids after trabeculectomy. Ophthalmology. 1995;102:1753-1759.

5. Giangiacomo J, Dueker DK, Adelstein E. The effect of preoperative subconjunctival triamcinolone administration on glaucoma filtration. I. Trabeculectomy following subconjunctival triamcinolone. Arch Ophthalmol. 1986;104:838-841.

6. Tham CC, Li FC, Leung DY, et al. Intrableb triamcinolone acetonide injection after bleb-forming filtration surgery (trabeculectomy, phacotrabeculectomy, and trabeculectomy revision by needling): a pilot study. Eye. 2006;20:1484-1486. 
